\title{
La información sobre Salud y Medicina en los diarios de la Comunidad Autónoma Vasca y Navarra
}

institucional.us.es/ambitos/

\author{
Alazne Aiestaran \\ Universidad del País Vasco \\ alazne.aiestaran@ehu.es \\ Idoia Camacho \\ Universidad del País Vasco \\ idoia.camacho@ehu.es \\ Milagros Ronco \\ Universidad del País Vasco \\ alazne.aiestaran@ehu.es
}

\section{Resumen}

Esta investigación analiza la cobertura periodística de los temas sobre salud y medicina en la prensa escrita diaria editada en la Comunidad Autónoma Vasca y en

la Comunidad Foral de Navarra desde 2001 hasta 2010. Se ha utilizado el método del análisis de contenido cuantitativo y cualitativo basado en la técnica de la semana compuesta, que nos ha permitido realizar un análisis diacrónico sobre el objeto de estudio. El estudio concluye que a pesar de haber aumentado la cantidad de informaciones sobre salud en los diarios analizados, los contenidos no profundizan en los temas tratados y carecen en la mayoría de los casos de enfoque educativo.

Asimismo, la información gráfica en la que se apoyan los textos se basa en

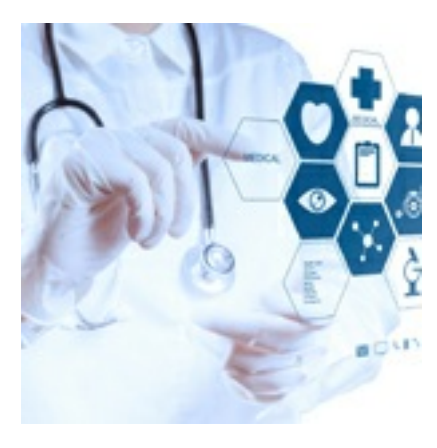

fotografías de poco valor informativo.

\section{Palabras clave}

Información sobre salud, medicina, prensa vasca, análisis de contenido.

\begin{abstract}
This research analyzes the media coverage of health and medical topics in the daily newspapers published in the Autonomous Community of the Basque Country and in the Chartered Community of Navarre from 2001 to 2010. We used the method of quantitative and qualitative content analysis based on the technique of composite week, which allowed us to carry out a diachronic analysis on the subject of study. The sample consists of the five

leading newspapers in both communities. The study concludes that,despite the fact that the analyzed newspapers have increased the amount of health related information,the contents do not go further into the researched issues and lack, in most cases, an educational approach. Furthermore, the graphical information in the texts is based on photographs of little informative value.
\end{abstract}

\section{Keywords}

Health information, medicine, Basque press, content analysis.

\section{INTRODUCCIÓN (1)}

La sociedad actual está cada vez está más concienciada en adquirir y mantener hábitos que contribuyan a mejorar su salud. Esta constatación está avalada por diversas investigaciones y estudios sociológicos que ponen de manifiesto que la salud y la medicina son uno de los temas que más interés suscita en la sociedad del siglo XXI. 
Barómetro Sanitario. En los últimos años, la salud destaca como uno de los temas que más inquieta a los ciudadanos por encima o a la par de otros tópicos como la educación, las pensiones o la vivienda.

En consecuencia parece lógico pensar que la salud es uno de los temas más buscados en internet. La encuesta realizada en 2010 por la empresa farmacéutica internacional Pfizer, llamada El rol de internet en el proceso de consulta de información sobre salud (2), indica que el $80 \%$ de los internautas españoles acude a la red para buscar información sobre temas de salud.

Este porcentaje pone a España al mismo nivel de Estados Unidos, donde, según el último informe sobre el tema emitido por el Centro de Investigación Pew, la salud continúa siendo -al igual que en la década de 2000uno de los temas más consultados en internet. Concretamente, el $80 \%$ de los usuarios de la red buscan información sobre salud online(Fox, 2011:1-2).

El incremento de la cantidad de informaciones sobre sanidad y medicina en los medios de comunicación responde al interés social sobre este tipo de cuestiones. En los grandes medios estadounidenses, el espacio dedicado a noticias sobre salud personal se multiplicó por cinco en dos décadas, según un estudio que analizó 16 medios distintos. En lo que a prensa escrita se refiere, el diario Los Angeles Times triplicó el número de noticias sobre salud y la revista TIME multiplicó por seis las portadas dedicadas a este tema (Tabakman, 2011: 2).

En España, el Informe Quiral es el referente anual sobre análisis de los temas de medicina y salud en la prensa escrita.Partiendo de los datos obtenidos para el citado informe,Revuelta y Minelli de Oliveira (Revuelta y Minelli de Oliveira, 2008: 64)determinaron que, "si bien la salud y la medicina ocupan un espacio considerable en la prensa diaria española, en realidad la información se estructura en torno a unos pocos temas, los cuales constituyen la actualidad del momento o, en otras palabras, la agenda social".

Pese a que el Informe Quiral es el trabajo más destacado en lo que respecta a la salud en prensa escrita, también existen otros trabajos de investigación de ámbito más local que ponen su punto de atención en los tópicos de salud. El estudioPrensa escrita y salud en Madrid analizó los temas de salud relacionados con Madrid publicados en 8 diarios de pago y gratuitos, (El País, El Mundo, ABC, La Razón, 20 Minutos, Metro, Qué y $A D N$ ). Según esta investigación, los diarios gratuitos publican más informaciones sobre salud que los de pago, aunque, entre los de pago, El País y El Mundo alcanzan el mismo nivel que los gratuitos (Ugarte, Menéndez y Cuesta, 2009: 113).

Costa (2008) analizó las piezas sobre medicina y salud publicadas por los cuatro diarios gallegos de mayor difusión. Una de las conclusiones de su estudio es que, aunque la prensa dedica un espacio importante a las noticias sobre salud -más de una página diaria de media- en general son textos cortos que no permiten contextualizar ni profundizar en la información.

Camacho, Peñafiel y Ronco (2012), en su investigación La información de salud en la prensa diaria vasca (2001-2010), concluyen que, a la vez que se aumentaba el número de informaciones sobre salud publicadas, en los últimos años ha habido un esfuerzo por parte de los medios de comunicación españoles, concretamente de la prensa, por mejorar la calidad de dichas informaciones, fundamentalmente, contratando a periodistas especializados en ese tema. Sin embargo, hoy en día la tendencia en los medios es a no invertir en especialización, lo que -unido a las prisas y a la inmediatez que imperan en el periodismo- hace mermar la calidad de las informaciones, por lo que es más fácil caer en el alarmismo y en crear falsas expectativas.

También existen varios trabajos sobre periodismo científico y la información de la ciencia en los medios de comunicación que hacen referencia a la salud desde la perspectiva del periodismo especializado, la divulgación y la calidad. Citemos, a título meramente ilustrativo los trabajos realizados por Carlos Elías (2002a), (2002b), (2003), Sergi Cortiñas (2007) y Carolina Moreno (2003).

Pese a que se ha constatado el aumentado del volumen de textos en torno a la salud en la prensa diaria, ese incremento en la oferta de contenidos sobre salud y medicina no siempre ha ido acompañado de una mejora esencial en su calidad, y aún queda mucho recorrido por andar para liberarnos de algunas prácticas periodísticas que siguen generando efectos contraproducentes en la ciudadanía (Observatorio de la 
Lo cierto es quelos medios de comunicación tienen un papel fundamental como intermediarios al interpretar las investigaciones médicas e informar sobre ellas en un lenguaje más accesible, de manera que sean más comprensibles para los ciudadanos (Viswanath y otros, 2008: 759) y al mismo tiempo se cree una mayor conciencia pública sobre la actividad científica (Nelkin, 2001: 199).

Las noticias sobre salud pueden implicar efectos negativos sobre la población, si no son tratadas con el suficiente rigor y la calidad necesaria en un tema tan crucial para el bienestar de una sociedad como es su propia salud. Se ha demostrado que la prensa condiciona la percepción que tiene el público sobre la gravedad de las enfermedades y que una información más objetiva y pedagógica sobre las dolencias contribuye a unamayor comprensión de las mismas por parte de la población (Young, Norman y Humphreys, 2008).

\section{METODOLOGÍA}

La investigación,pionera en los medios de comunicación vascos dado que hasta ahora no se han llevado a cabo estudios sobre la temática de salud, tiene como objetivo analizar la cobertura periodística de los temas de salud en la prensa escrita diaria editada en la Comunidad Autónoma Vascay en la Comunidad Foral de Navarra desde el año 2001 hasta el 2010.

La hipótesis de partidaes que en la primera década del siglo XXI la información sobre salud en la prensa vasca y navarra se ha incrementado y ha conseguido afianzarse en la agenda periodística de los diarios. Sin embargo, la salud es un campo que tiene limitados recursos dentro de las ediciones de los diarios, hecho que incide de manera negativa en el producto final. También toma fuerza la idea de que la mayoría de las piezas publicadas no se abordan desde una perspectiva educativa para con los lectores.

Con la finalidad de dar respuesta a todas estas preguntas, en el análisis se han empleado técnicas de metodologías cuantitativa y cualitativa. Entre las técnicas de investigación cuantitativa se ha utilizado el método del análisis de contenido, que ha hecho posible estudiar los textos publicados de una forma sistemática, objetiva y cuantitativa (Wimmer y Dominick, 1996). Hemos consideradopertinente el análisis de contenido porque "se mueve entre dos polos: el rigor de la objetividad y el de la fecundidad de la subjetividad" (Bardin, 2002: 7).

La investigaciónha llevado a cabo un análisis diacrónico de la evolución de los temas de salud en los diarios de referencia de las dos comunidades autónomas durante el periodo de 10 años. Para ello,se ha utilizado como base la técnica de la semana compuesta, técnica reconocida y probada por varios autores entre los que destacan Riffe, Aust y Lacy, Stempel (Zabaleta, 1997: 199-203). Estos investigadores compararon para ello tres tipos de muestra: la semana simple, la semana compuesta y la semana continua. $Y$ en cada una de ellas aplicaron cuatro tipos de tamaño de intervalo: 7, 14, 21, 28 días. La semana compuesta obtuvo una mayor fiabilidad que las otras muestras (Echegaray y Ayestaran, 2012: 11).

Para la extracción de la muestra se ha seleccionado el modelo de semana compuesta, que se ha aplicado para cada uno de los años a analizar. Según Zabaleta, para el análisis de contenido de un año en prensa la muestra idónea es de dos semanas compuestas, es decir de 14 días (Zabaleta, 1997: 199). En esta investigación, hemos ampliado esa muestra idónea, y analizado 21 días cada uno de los 10 años estudiados.

La muestra la componen las cinco cabeceras de referencia de la Comunidad Autónoma Vasca y Navarra, por ser las que llegan al mayor número de personas y por recoger las diferentes posturas ideológicas: por un lado se han escogido los diarios El Correo, Noticias de Gipuzkoa y Noticias de Álava que pertenecen a los dos grupos mediáticos lideres en Euskadi. Por otro lado, se ha seleccionadoDiario de Navarra que es el periódico decano en la Comunidad Foral de Navarra y Berria por ser el único diario que se publica íntegramente en euskara en las dos comunidades autónomas.

Hay que indicar que no todos los diarios analizados tienen una antigüedad anterior al año 2001, año en el que comienza el estudio. Berria, Noticias de Álava, Noticias de Gipuzkoa han hecho su aparición en el panorama mediático en 2003, 2004 y 2005 respectivamente. El primer número de Berria se publica el 21 de junio de 2003 como continuación del clausurado Euskaldunon Egunkaria (3). Por lo tanto, durante los dos primeros años de 
estudio se ha analizado Euskaldunon Egunkaria, hasta su desaparición, mientras que a partir de 2003 entra en la muestra Berria, considerado como la sucesión del anterior. El 10 de noviembre de 2004 sale al mercado el primer número de Noticias de Álava y el 24 de noviembre de 2005 se publica Noticias de Gipuzkoa, ambos pertenecientes al Grupo Noticias.

La unidad de análisis para esta investigación la ha formadotodo texto informativo, interpretativo y de opinión relacionado directamente con temas de salud. Se han sometido a examen los textos en su totalidad (título, subtítulo, antetítulo, cintillo y texto completo).

También se ha utilizado la técnica de la entrevista en profundidad, sobre todo por la capacidad que posee para obtener información cualitativa. De hecho,"la importancia de las entrevistas en profundidad estriba enque permiten conocer a la gente lo bastante bien como para comprender lo que quieredecir, y crean una atmósfera en la cual es probable que se exprese libremente" (Taylor y Bogdan, 1987: 199).

Además, frente a otras técnicas cualitativas de investigación como la observación "la entrevista en profundidad es más capaz y eficaz en el acceso a la información difícil de obtener sin la mediación del entrevistador o de un contexto grupal de interacción" (Vallés, 1999: 196-197).

En estecaso, las entrevistashan sido necesarias e imprescindibles para verificar, interpretar y completar la información extraída de los datos del análisis de contenido. Las entrevistas en profundidad se realizarona los periodistas encargados de redactar los textos de salud en cada diario analizado y se llevaron a cabo de modo presencial entre julio, agosto y septiembre de 2012 (4).Se confeccionó un modelo de cuestionario que contenía un total de 43 preguntas divididas en tres apartados. El primer bloque estaba compuesto por 13 preguntas sobre elfuncionamiento del periódico en relación a las informaciones de salud, la segunda parte tenía 19 preguntas relacionadas con los contenidos de salud en el periódico y el último apartado, de 11 preguntas, abordaba aspectos generales sobre el binomio periodismo/salud.

\section{RESULTADOS}

\subsection{Desarrollo de los temasde salud en la prensa vasca y navarra}

Se han registrado 3.311 textosen la muestra elegida durante el periodo de 10 años. La evolución de los textos a lo largo de ese tiempo indica un aumento de los contenidos relacionados con la salud en la prensa vasca y navarra a partir de 2004 hasta llegar a su punto álgido en los años 2006 y 2007.

Gráfico 1. Desarrollo del núm ero de piezas sobre salud

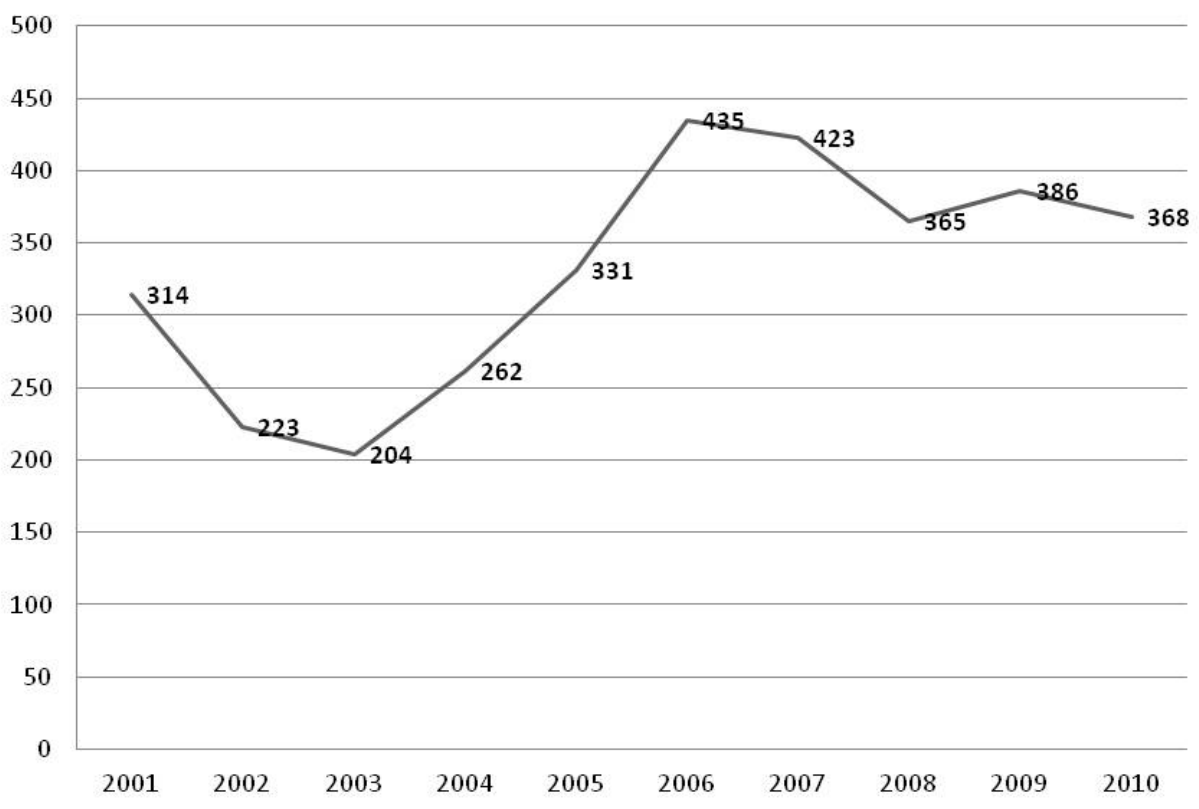

Fuente: Elabora ción propia a partir de los datos ex traídos de la investigación 
La razón de ese incremento es clara: por un lado, en 2005 están en el mercado todos los diarios que componen la muestra y, por otro lado, todavía el sector no se encuentra inmerso en la crisis económica mundial que comenzó en 2007-2008 y en la que todavía hoy España sigue sumida. Además, como señalan los periodistas entrevistados, la salud es uno de los temas que más interesa a la sociedad en general y eso se ha reflejado en los diarios, donde se les ha dedicado más espacio.

Uno de los criterios que se utilizan a la hora de seleccionar las informaciones suele ser precisamente el interés público. "Son noticias que derivan de la necesaria convivencia de las personas en el marco de una sociedad. De ahí que hagan referencia a temas comunes como político, social, asociativo, etc." (Armentia y Caminos, 2009: 220).De hecho, la mayoría de los diarios analizados publican suplementos que, aunque no en todos los casos son monográficos de salud, muchos temas relacionados con la sanidad encuentran su sitio en los mismos.

A partir de 2007se aprecia un decrecimiento paulatino del número de noticias, como consecuencia de la crisis económica. Por un lado, todos los diarios disminuyen el número de páginas general del periódico, lo que repercute directamente a las páginas dedicadas a las diferentes secciones. Por otro lado, Berria y Noticias de Gipuzkoa dejan de editar los suplementos dedicados a varios temas entre los que se encuentran los tópicos de salud. En la prensa vasca y navarra, como en la prensa de España, tampoco existe una dirección clara respecto a los suplementos aunque la crisis ha afectado directamente al número de páginas (Observatorio de la Comunicación Científica, 2008: 14).

Al igual que ha sucedido con otras temáticas como la ciencia, hoy en día la mayoría los suplementos y secciones sobre salud han desaparecido o han sido reducidos a la mínima experesión (Cortiñas, Lazcano y Pons, 2013: 79). La crisis ha sido uno de los factores más decisivos para que los suplementos desaparezcan o disminuyan sus páginas.Así lo constatan todos los periodistas entrevistados.Fermín Apezteguia, periodista que se ocupa en El Correode la información referida a la salud, apunta a la recesión económica como uno de los factores que ha afectado al descenso de los contenidos sanitarios.

La actual situación de crisis económica ha llevado a los medios de comunicación a reducir sus plantillas y limitar el espacio reservado para todo tipo de información. Ese espacio está regulado por ley y la publicidad manda. A más publicidad, más espacio. A menos ingresos publicitarios, menos espacio para todas las secciones y menos manos para trabajar. No ha bajado la calidad de las informaciones -al contrario, personalmente creo que ha subido-, pero sí la cantidad (5).

Los periódicos que durante el periodo de investigación han publicado más piezasrelacionadas con la saluden valores absolutos coinciden con aquellos que más tiempo llevan en el mercado, Diario de Navarra seguido de El Correo, como se puede apreciar en la tabla1. 


\begin{tabular}{|l|c|c|}
\hline Diario & Total & Porcentaje \\
\hline Diario de Navarra & 777 & $23,50 \%$ \\
\hline El Correo & 706 & $21,30 \%$ \\
\hline Noticias de Gipuzkoa & 604 & $18,25 \%$ \\
\hline Noticias de Álava & 506 & $15,26 \%$ \\
\hline Berria & 471 & $14,24 \%$ \\
\hline Egunkaria & 247 & $7,45 \%$ \\
\hline Total & $\mathbf{3 . 3 1 1}$ & $\mathbf{1 0 0 \%}$ \\
\hline
\end{tabular}

Fuente: Elaboración propia a partir de los datos ex traídos de la investigación

No obstante, la evolución de cada diario por años no marca la misma tendencia. En proporción Noticias de Gipuzkoa y Noticias de Álava, que llevan mucho menos tiempo en el mercado, han publicado más piezas que las cabeceras más antiguas.

A partir del cierre de Euskaldunon Egunkaria se publica Berria, que -excepto en 2004- tiende a disminuir los contenidos de salud con respecto a su predecesor. Diario de Navarra es líder en información sobre salud durante los años 2003 y 2004 , pero en2005 comienza un descenso acusado del número de piezas. El Correo no destaca ningún año por ser quien más textos publica, y su número desciende a partir de 2007. En el caso de Noticias de Álava y Noticias de Guipuzkoa, llama la atención el incremento de loscontenidos de salud durante los siguientes cuatro años. De hecho, Noticias de Gipuzkoa encabeza el ranking de contenidos desde 2006 hasta 2010.Según el periodista de Noticias de ÁlavaCarlos Martínez, el volumen de la información sobre salud ha sido bastante uniforme, tanto en cantidad como en calidad, dado que siempre se le ha dado importancia y ha tenido relevancia en el periódico.

\subsection{Temas de mayor relevancia}

La diversidad es la característica principal de los temas de salud publicados en la prensa vasca y navarra durantelos 10 años de estudio. La mayoría de las cuestiones no suponen más de un $2 \%$ del total. Es decir, un total de menos de 50 piezas durante todo el periodo de análisis. En la siguiente tablase indica cuáles han sido los diez temas que más relevancia han tenido durante este intervalo de tiempo: 
Tabla 2. Tem asprincipales

\begin{tabular}{|l|c|}
\hline Temas & Total \\
\hline Enfermedades infecciosas & $10,54 \%$ \\
\hline Atención sanitaria & $8,64 \%$ \\
\hline Enfermedades psicológicas & $6,10 \%$ \\
\hline Investigación & $5,59 \%$ \\
\hline Legislación y política sanitaria & $5,01 \%$ \\
\hline Sexualidad y reproducción & $4,68 \%$ \\
\hline Colectivo sanitario & $4,44 \%$ \\
\hline Cáncer & $4,14 \%$ \\
\hline Hospitales y Centros de Salud & $3,84 \%$ \\
\hline Alimentos (en relación a la salud) & $3,59 \%$ \\
\hline
\end{tabular}

Fuente: Elaboración propia a partir de los datos ex traídos de la investigación

Entre los diez temas clasificados en la tabla se distinguen dos grandes grupos: los temas relacionados con los contenidos vinculados a la medicina y la salud, y los que están ligados a la actualidad informativa.

Entre las materias relacionadas directamente con la medicina y la salud sobresalen las enfermedades infecciosas, que se sitúan en la primera posición de la tabla. Dentro de las piezas sobre estas enfermedades, casi la mitad, un $44 \%$, lo ocupan las piezas relaciones con el sida. Dentro del $56 \%$ restante las enfermedades infecciosas que más presencia han tenido han sido las siguientes, en este orden: la gripe A, la gripe aviar, la enfermedad de las vacas locas, y la legionela. Los datos revelan que además de ser los tópicos más repetidos son también los que han conseguido ser portada entre las enfermedades infecciosas.

Este tipo de cuestiones responden a las características del patrón agudo o, lo que es lo mismo, sonasuntos de interés súbito (Observatorio de la Comunicación Científica, 2010: 42).Suelen ser temas como brotes epidémicos, intoxicaciones alimentarias... que atraen mucho interés pero por muy poco tiempo y tienden a ocupar más portadas que otros temas. En este caso, del total de piezas analizadas, tan solo un $10,6 \%$ ocupan portada.

Los trastornos psicológicos suponen el $11 \%$ de las enfermedades que con mayor frecuencia han aparecido en las páginas de los diarios. El Alzheimer es, sin lugar a dudas, el trastorno más recurrente. La razón principal es que el 21 de septiembre, Día Mundial de la enfermedad, es uno de los días que componen la muestra elegida para el análisis. De hecho, en el cómputo general de todos los diarios el mes que más noticias sobre esta enfermedad concentra es septiembre. Además, dentro del apartado de enfermedades psicológicas ocupan un lugar preferente la anorexia, la bulimia, la depresión, la ansiedad, las adicciones y los trastornos de conducta.

Inmediatamente después de los trastornos psicológicos se sitúan los textos sobre investigación, dentro de los cuales la diversidad es la característica principal. De todos modos, las cuestiones que más abundan son las investigaciones en torno a la genética, las células madre, el cáncer, la clonación y el sida.

Dentro del apartado de sexualidad y reproducción,destacan los textos dedicados a tratar cuestiones como los beneficios de la sexualidad para la salud, la reproducción asistida, fecundación in vitro y la píldora postcoital. En el caso de los beneficios que la sexualidad tiene en la salud, todos los textos codificados pertenecen al diario Berria, que todos los martes cuenta con la colaboración de personal sanitario, entre ellos una sexóloga, que plantea diversas cuestiones en torno a la sexualidad.

El cáncer es otro de los temas con presencia importante en todos los diarios. Pese a que la enfermedad abarca 
diversos tipos de tumores, algunos de ellos son tratados con mayor frecuencia. El cáncer de mamá es el que más atención concentra en los diarios, seguido de tópicos como la prevención del cáncer, cáncer de pulmón, próstata o cáncer de piel. Estos datos coinciden con los presentados por el Informe Quiral 2011, según el cual, además de la gravedad de la enfermedad, existen otros factores para que los medios se fijen en ella, como el tipo de población a la que afecta, si el tema produce novedades, la efectividad de los esfuerzos comunicativos de las fuentes emisoras de la información como laboratorios, farmacéuticas, universidades, hospitales, etc. (Observatorio de la Comunicación Científica, 2012: 17).

En último lugar de la lista de los diez temas más frecuentes, aparecenlas cuestiones relacionadas con los alimentos. En este apartado los argumentos más repetidos son los referentes a intoxicaciones, alimentos contaminados y alimentos transgénicos. Este tipo de informaciones también entran dentro de los temas de patrón agudo.

Gráfico 2. Los 10 tem as destacados enla prensa vasca ynavarra

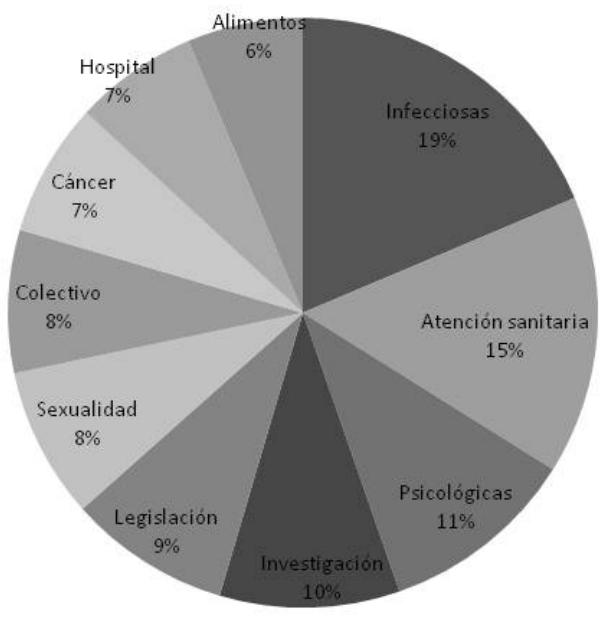

Fuente: Elaboración propia a partir de los datos ex traídos de la investigación

Cuatro de los temas que figuran en el gráfico anterior están ligados a la actualidad informativa más que a la medicina. El primero de esos temas es la atención sanitaria, que es el segundo tema en cuanto a porcentajes en el gráfico y que coincide con algunas de las valoraciones hechas por los periodistas entrevistados para esta investigación. La mayoría de los profesionales definen la atención sanitaria como un tema de interés general, es decir, uno de los temas que más preocupa a la ciudadanía. Como consecuencia, aparece muy a menudo reflejado en las informaciones del día a día en los diarios.

Otro de los temas relacionados con la actualidad es el de legislación y política sanitaria, tópico recurrente que suele aparecer en piezas elaboradas con información y opinión proveniente de partidos políticos y sindicatos, además de asociaciones profesionales de médicos, enfermeras etc. El tema de legislación sanitaria aparece vinculado a argumentos como la despenalización del aborto, la opción a elegir una muerte digna, la Ley de la Dependencia o la Ley del Tabaco.

En tercer lugar, aparecen los asuntos relativos al colectivo sanitario. En este apartado, las informacionesmás numerosas sonlas que se refieren a ofertas de empleo público, y a movilizaciones y huelgas delpersonal sanitario por reivindicaciones laborales.

Hospitales y centros de salud es la otra temática ligada a la actualidad informativa que aparece de manera continuada en todos los diarios.Dentro de ella destacan los textos sobre construcción y/o remodelación de edificios de hospitales, clínicas, centros de salud, residencias de ancianos y geriátricos. 
Entre los diarios analizados no existen diferencias llamativas en los asuntos relacionados con el día a día. El número de ítems publicados de acuerdo con esos argumentos es muy parecido en todos los periódicos analizados.

\subsection{Ubicación y géneros periodísticos utilizados}

Los datos generales muestran que la salud no es un argumento de portada, no al menos en los últimos 10 años en la prensa diaria analizada en este trabajo. Sólo un 10,62\% de los contenidos revisados aparecen en portada. Asimismo, la relevancia de los argumentos tampoco se refleja en la apertura de sección, ya que sólo un 13,76\% de los textos analizados abren la sección.

Tabla 3. Tem as de salud en portada

\begin{tabular}{|l|c|c|c|}
\hline Diarios & No & Sí & Total \\
\hline El Correo & 668 & 38 & 706 \\
\hline Diario de Navarra & 663 & 114 & 777 \\
\hline Noticias de Gipuzkoa & 519 & 85 & 604 \\
\hline Noticias de Álava & 441 & 65 & 506 \\
\hline Berria & 425 & 46 & 471 \\
\hline Egunkaria & 243 & 4 & 247 \\
\hline Total & & & \\
\hline
\end{tabular}

Fuente: Elaboración propia a partir de los datos ex traídos de la investigación

Los datos de la tabla 3 están estrechamente ligados a los géneros que predominan en la prensa vasca y navarra para presentar los tópicos de salud. El género más utilizado es precisamente el informativo, tal y como se puede apreciar en el siguiente gráfico. 


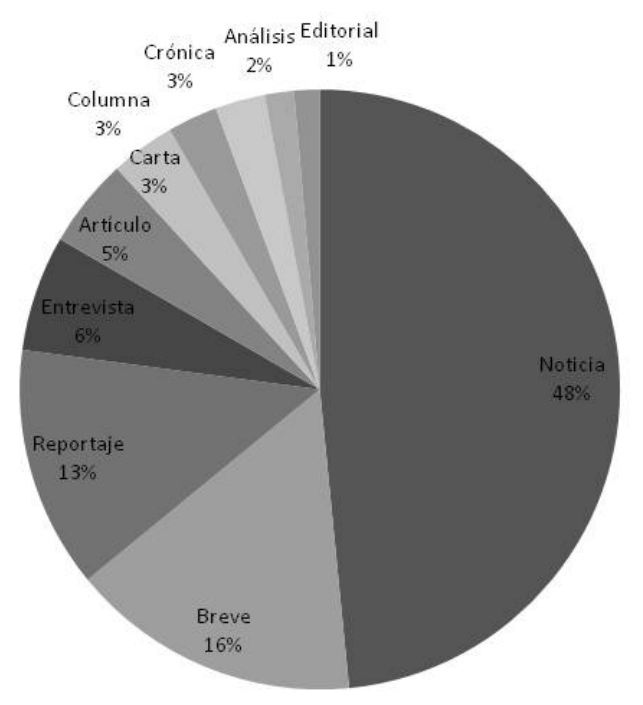

Fuente: Elaboración propia a partir de los datos ex traídos de la investigación

Más de la mitad del total de los textos revisados (2.083) son noticias y noticias breves. Se trata de géneros informativos puros, que -además de estar muy ligados a la actualidad diaria- no permiten profundizar de manera exhaustiva en el tema. "Los géneros estrictamente informativos, dada su rigidez, presentan el inconveniente de que pueden no ayudar lo suficiente a motivar el lector ni permiten explotar la creatividad del divulgador" (Cortiñas, 2007: 374). Los géneros de interpretación que posibilitan ahondar más en los temas no tienen tanta presencia como los informativos. De hecho, entre los géneros interpretativos destaca el reportaje, con un total de 424 piezas publicadas, y en menor medida la entrevista, que se publica en 203 ocasiones. Entre los géneros opinativos, el más utilizado es el artículo de opinión, del que se han contabilizado 157 piezas.

En lo que se refiere a la ubicación de los textos sobre salud, como se aprecia en el siguiente gráfico, la mayoría encuentran su espacio en la sección de Sociedad (43\%), y en la sección de Local (20\%). En menor medida, las cuestiones relacionadas con la salud aparecen en secciones como opinión (9\%), Estado (6\%) o Ciencia (5\%).

Estas cifras-similares a las que han obtenido otros estudios, como el de Cortiñas(2007), evidencian que los contenidos sobre salud y medicina no cuentan en los periódicos con una relevancia suficiente que les conceda tener una sección propia, "por lo que se aplica un criterio a medio camino entre el cajón de sastre de Sociedad y el ámbito geográfico de la información”(Costa, 2008: 18). 
Gráfico 4. Secciones en las que se ubicanlos textos

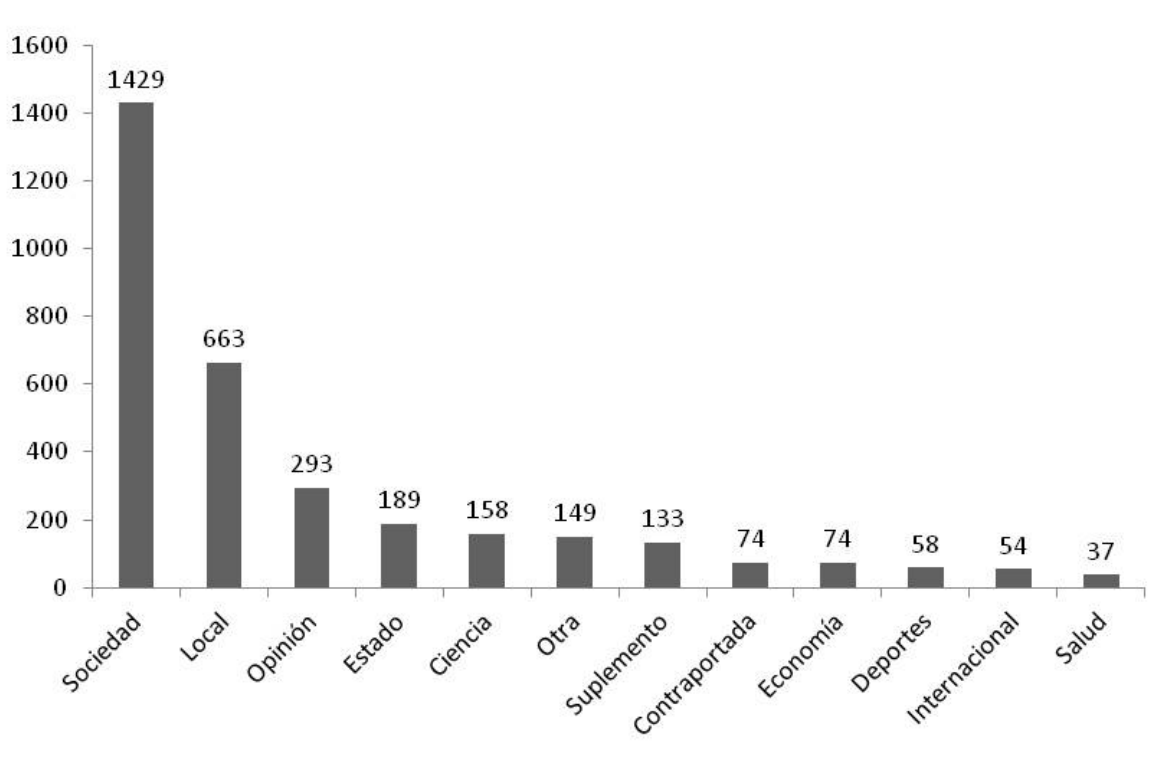

Fuente: Elaboración propia a partir de los datos ex traídos de la investigación

\subsection{Firmade los textos}

Casi la mitadde las piezas analizadas están firmadas por redactores de los diarios (43\%). Otras investigaciones que también analizan las noticias de salud, como el Informe Quiral 2009 o la llevada a cabo por Costa (2008), corroboraneste dato, ya que en los diarios de referencia la mayor parte de los contenidos están elaborados por los periodistas que pertenecen a la plantilla.

Los profesionales entrevistadospara este estudio admiten no tenerespecialidad de divulgación científica o de salud, pese a que llevan una media de 6 años cubriendo temas de sociedad y-dentro de esta sección- los argumentos de salud. A pesar de esta falta de formación adicional en sanidad, todos coinciden en que tienen un interés personal en contenidos de salud y la sanidad. Además, varios de ellos han considerado que la experiencia de años trabajando en el ámbito de la salud ya les ha dado una cierta especialización o, al menos, un amplio conocimiento del tema. "Lo habitual en España es que la especialización se adquiera abordando acontecimientos informativos de temática similar, ya sea en el ámbito de la sanidad, la educación, la ciencia, la política, la religión o el deporte." (Elías, 2002a: 391). 


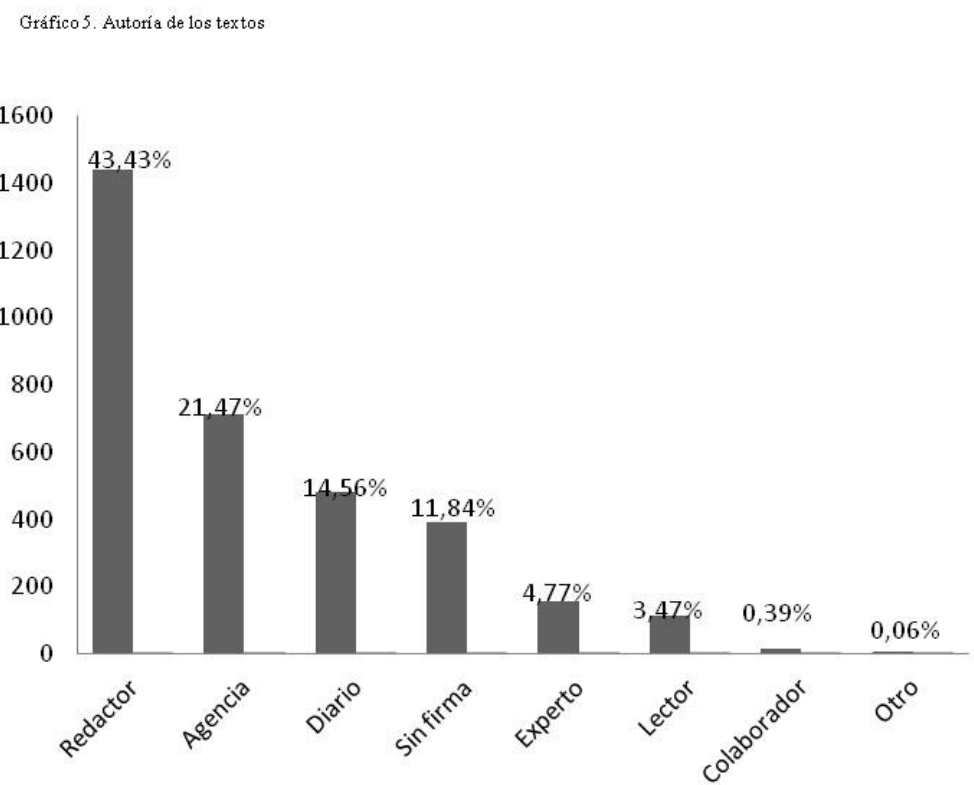

Fuente: Elaboración propia a partir de los datos extraídos de la investigación

Entre los redactores que firman las noticias de salud hay una gran diversidad de perfiles. Los que más piezas escriben en cada diario son los que nombramos a continuación. En Diario de Navarra las firmas más destacadas son las de María Jesús Echeverría y María Jesus Castillejo. En El Correo aparecen como periodistas escribiendo sobre temas de saludFermín Apezteguía, Carmen Barreiro y Marta Fernández Vallejo. EnEuskaldunon Egunkaria y Berria los nombres más frecuentes son Arantxa Iraola, Ainara Arratibel y Edurne Elizondo. En Noticias de Álava destacan Carlos Martínez Orduna y Nekane Lauzirika. Jorge Nepal y Ana Úrsula Soto son los periodistas que más textos firman en Noticias de Gipuzkoa.

Ningún periodista de los nombrados anteriormente está especializado exclusivamente en temas de salud, ya que la mayoría pertenece a la sección de sociedad y elabora todo tipo de temas que tienen cabida en ese apartado del diario.

Las agencias se sitúan en segundo lugar en cuanto a número de piezas firmadas, por delante de la firma colectiva con el nombre del diario. Es decir, un $22 \%$ del totalde lostextos revisados, lleva el nombre de agencias como EFE y Colpisa o la alemana DPA para los casos de carácter concreto e internacional en el caso de Diario de Navarra. En El Correo, al igual que en Euskaldunon Egunkaria y Berria, en la mayoría de las ocasiones no se especifica el nombre de la agencia, y en los casos que se precisa se trata de las agencias Colpisa y EFE. En Noticias de Álavaprevalecen EFE y Europa Press. A pesar de pertenecer al mismo grupo de comunicación, en Noticias de Gipuzkoa destaca la presencia de EFE y son muchas las piezas que no especifican el nombre de la agencia.

Llama la atención el reducido número de piezas que están firmadas por personas expertas en diferentes áreas de salud. En general hay pocas diferencias entre las cinco cabeceras analizadas, dado que los textos elaborados por profesionales de la sanidad son artículos de opinión y columnas. Sin embargo, en el caso de Berriase aprecia un esfuerzo por introducir temas de salud una vez por semana. Para ello cuentan con la colaboración habitual de expertos en medicina (Jabier Agirre e Iñaki Arratibel, médicos), en sexualidad (Agurtzane Ormatza), en medicina alternativa (Nekane Martiarena, Eusebio González),psicólogos (Arantza Quecedo) y nutricionistas (Arantza Lorenzo). Esto hace que durante los diez años de análisis Berria sea en valores absolutos el periódico con más piezas firmadas por expertos en salud.

\subsection{Perspectiva educativa}

Al analizar cada información, hemos considerado que tenía una orientación educativa si incidía en cómo mejorar el cuidado de la salud o en la prevención de las enfermedades o, en general, si incluía acciones para 
adquirir hábitos y estilos de vida más saludables. Podemos concluir que las piezas analizadas no están elaboradas desde una perspectiva educativa. Este dato coincide con los resultados expuestos por Costa (2008), según los cuales, en las noticias sobre salud de los diarios de referencia de Galicia, son muy pocas la piezas divulgativas cuyo objetivo es incrementar el conocimiento de lector con una función formativa más allá de la mera información, en ocasiones de corte sensacionalista y muy sujeto a temas de agenda política.

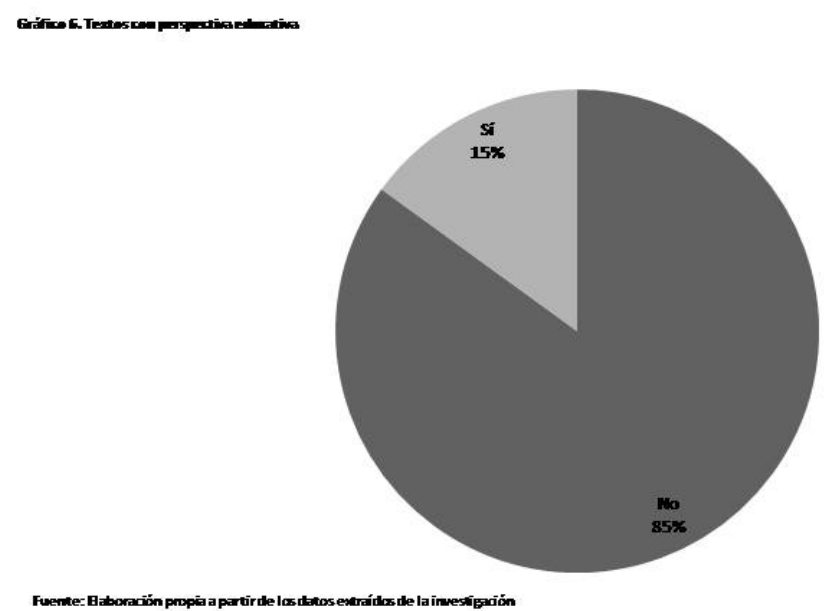

El número de textos que no incluye una perspectiva educativa o de formación (2.814) es casi seis veces mayor al número de textos que sílo hace (497). Este dato pone de manifiesto que en los temas principales que hemos destacado en el apartado anterior no encontramos la faceta de educación en salud. Nos referimos a temas como política y legislación sanitaria, centros hospitalarios, conflictos laborales, ofertas de empleo público, comunicaciones de la Administración, políticos y sindicatos, que están más ligados a la actualidad informativa y a la política sanitaria y que en muchas ocasiones se presentan en géneros de pura información (noticias o noticias breves).

Sin embargo, en las entrevistas realizadas a los periodistas de los medios analizados concuerdan las opiniones respecto a este tema. Todos y todas inciden en que hay un esfuerzo por parte de los medios y de los periodistas para elaborar textos con contenido educativo.

Maria José Etxeverria, periodista de Diario de Navarra, señala la importancia de utilizar infografías y desglosar bien la información de salud. Ainara Arratibel, de Berria, subraya la importancia de ofrecer testimonios de personas afectadas. En el caso de Berria, cabe señalar que los artículos y reportajes elaborados por profesionales del campo de la medicina general, deportiva, nutrición y psicología tienen una clara función educativa.

Fermín Apezteguia,de El Correo, apunta la apuesta por el desarrollo de una información en la que la prevención ocupa un papel fundamental y como prueba de ello se refiere a la puesta en marcha hace cinco años, en colaboración con la agencia de comunicación Docor, del programa Encuentros con la Salud de El Correo, para la prevención, formación y divulgación científica, que consiste en la organización mensual, de octubre a junio, coincidiendo con el año escolar, de nueve charlas dobles sobre un tema de salud de actualidad.

En opinión de Ana Úrsula Soto, del diario Noticias de Gipuzkoa,el periodista que se dedica a cubrir información sobre salud sabe y es consciente de la responsabilidad que conlleva como profesional y como persona elaborar ese tipo de información que puede influir en las decisiones de la gente. Carlos Martínez, de Noticias deÁlava, apela a las fuentes de información y opina que cuanto mayor sea el número de fuentes mejor se puede incidir 
en la perspectiva educativa y de divulgación. En su opinión, para ello se necesita más tiempo, pero cada vez hay menos tiempo para elaborar las informaciones.

La sobrecarga de trabajo en las redacciones es otra de las consecuencias de la crisis, que obliga a realizar el mismo trabajo con menos personal, hecho que puede tener consecuencias negativas en la calidad informativa, como han demostrado otras investigaciones. "Esta sobrecarga tiene efectos en la calidad informativa, pues las rutinas de producción, investigación y elaboración de cada pieza periodística se deben realizarigual, pero en menos tiempo" (Cortiñas, Lazcano y Pont, 2013: 84)

\subsection{Fuentes de información}

Más de la mitad de las fuentes de información contabilizadas en la investigación son fuentes institucionales, que en gran medida están relacionadas con los temas que más presencia han tenido durante el periodo de análisis y que hemos clasificado en dos bloques.

Por un lado, en los tópicos vinculados a la actualidad informativa prevalecen las fuentes institucionales, como instituciones políticas (Diputaciones, Gobiernos, ayuntamientos, consejerías, partidos políticos y sindicatos) e instituciones médicas (hospitales, Servicio vasco y navarro de salud, Organización Mundial de Salud y empresas sanitarias). Este dato corrobora la tendencia de otras investigaciones, dado que las fuentes de instituciones públicas aparecen entre las más utilizadas por los periodistas especializados en la producción de noticias y reportajes científicos (Moreno, 2010: 121).

Por otro lado, en las cuestiones relacionadas directamente con la salud y la medicina los diarios acuden a los expertos, médicos e investigadores en su mayoría, que constituyen la segunda fuente más utilizada por los medios vascos. Como apunta el Informe Quiral, el periodista busca en las fuentes institucionales la voz del poder oficial, mientras que en los expertos busca la credibilidad y la opinión del saber experto(Observatorio de la Comunicación Científica, 2008: 17).

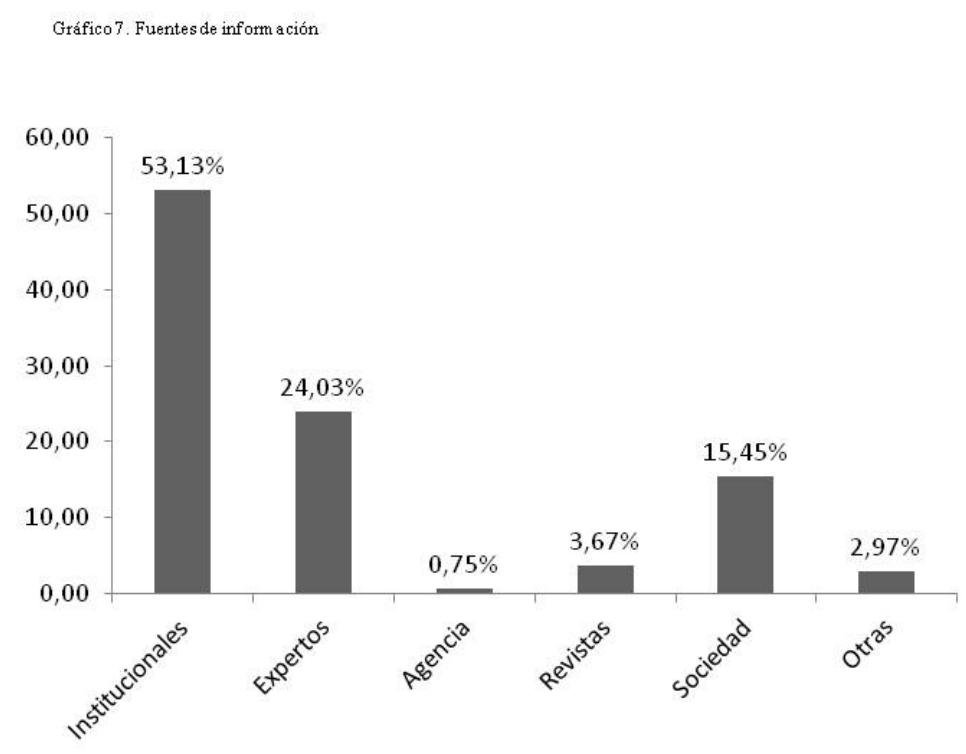

Fuente: Elaboración propia a partir de los datos ex traídos de la investigación

Otra de las fuentes de información que tiene relevancia para los diarios vascos y navarros es la sociedad civil, como se puede apreciar en el gráfico. En este colectivo, los periodistas acuden sobre todo a personas enfermas o sometidas a tratamiento médico, o a asociaciones de enfermos. Este dato también se corrobora en el informe Quiral, que destaca el aumento de la presencia de este sector a lo largo de los años (Observatorio de la Comunicación Científica, 2008: 17). 
Respecto a las revistas científicas, cabe señalar que se han citado como fuente en 440 ocasiones en los cinco periódicos estudiados. Las revistas más mencionadas son aquellas que gozan de mayor prestigio en la comunidad científica, como Nature, The Lancet, Science,New England Journal of Medicine, New Scientist o British Medical Journal, entre otras. Estas mismas revistas son las más citadas en estudios realizados por el Observatorio de Comunicación Científica de Barcelona (Elías, 2002b: 124) o el llevado a cabo por Carlos Elías (2002b) donde analiza la influencia de las revistas de impacto en el periodismo científico y en la ciencia actual. El autor interpreta que en el periodismo científico español sucede lo mismo que en otros países. Es decir, que las revista científicas más citadas y de más prestigio para los científicos son las que más aparecen en los diarios.

Es importante destacar que el $22,75 \%$ de los 3.311 registros contabilizados no menciona ninguna fuente de información. De ese porcentaje, el $14 \%$ pertenece a piezas informativas (noticias breves y noticias) y de interpretación (reportajes y entrevistas) y el 8,55\% corresponde a los textos de opinión (editorial, artículo, columna o cartas al director) donde en la mayoría de los casos predomina la opinión y valoración del autor/a y no se citan fuentes.

Todos los y las periodistas entrevistados subrayan la dificultad para acceder a las fuentes oficiales como Osakidetza (Servicio Vasco de Salud), Osasunbidea (Servicio Navarro de Salud), Ministerios u Organización Mundial de la Salud de un modo directo, porque los periódicos en los que trabajan son medios que trabajan en un ámbito más reducido y local.

\subsection{Información gráfica}

En los textos sobre salud analizados en este trabajo se han publicado un total de 2.463 piezas gráficas. De todas ellas la gran mayoría son fotografías (1.863), seguidas a gran distancia de los gráficos (330) y de los dibujos (233).

Se puede observar el escaso uso de infografías (37) por parte de todos los periódicos, ya que es precisamente este tipo información gráfica la que permite explicar y profundizar en los temas que se tratan.

Gráfico 8. Im ágenesutilizadas

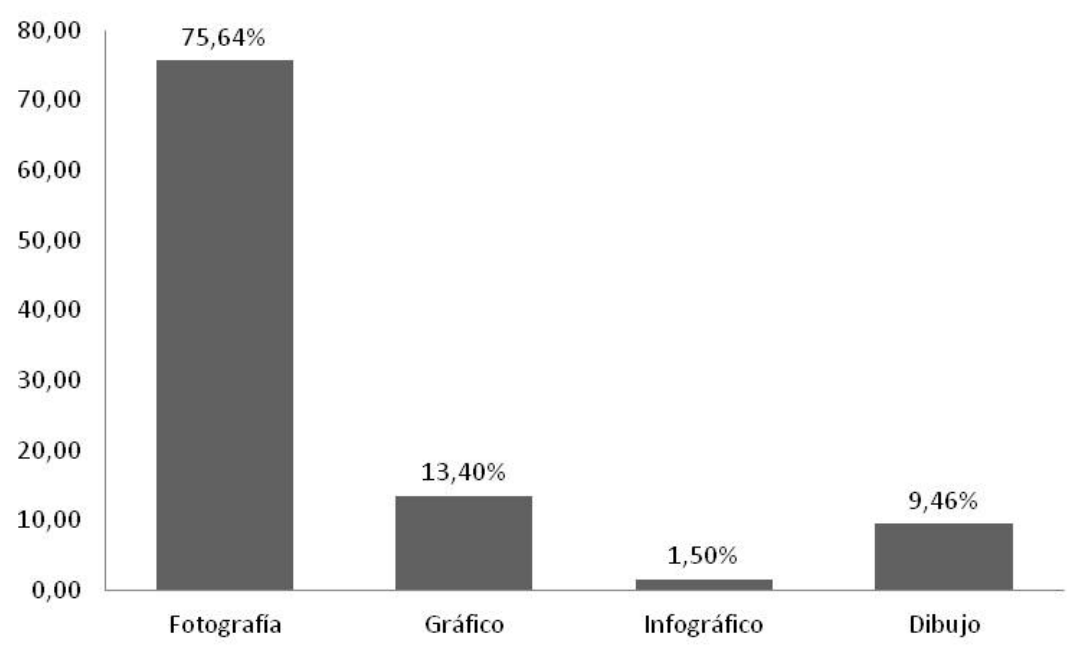

Fuente: Elaboración propia a partir de los datos extraidos de la investigación

Las imágenes, fotografías la gran mayoría, se utilizan sobre todocon el objetivo y la función de simple ilustración, no para aportar mayor información al texto escrito. Respecto a este tema, María José Echeverría periodista de Diario de Navarra-cree necesaria la aplicación de la ética y la deontología periodística. 
Por ejemplo si hacemos un reportaje sobre Alzheimer procuramos no poner una fotografía que salga una persona que se le ve deteriorada. En esos casos se suele utilizar una imagen en la que se ve a unas personas que se dan la mano o alguien atendiendo. Algo que no te encoja el corazón, que para eso, ya tienes otras cosas a diario, sobre todo si es una información divulgativa. Esos temas se cuidan bastante (5).

En lo que se refiere al restringido uso de gráficos e infografías, los periodistas entrevistados admiten que la infografía no se puede utilizar entodas las piezas de información que querrían, sobre todo porque resulta muy caro económicamente. Carlos Martínez - de Noticias de Álava- incide en los recortes que el diario ha sufrido. "Cuando empezamos sí que teníamos una infógrafa que se marchó al periódico de Pamplona y no fue sustituida. Ella apoyaba muy habitualmente con sus gráficos las informaciones de salud y muy bien, además. El motivo ha sido estrictamente económico" (6).

Por su parte, Fermín Apezteguia -de El Correo-también acepta que existen limitaciones para utilizar infografías, pero señala que su diario ha apostado por usar otros elementos estilísticos:

Infografía es una sección que da servicio al conjunto del periódico. Todas las secciones recurren a ella y, lógicamente, no da abasto para la demanda que recibe, a pesar de que lo intenta. Como es imposible que lleven gráficos todas las noticias que querríamos, se han desarrollado otros elementos estilísticos de diseño que nos ayudan a desmenuzar la información y darla más digerible: fichas explicativas, negritas con las ideas clave, sumarios que destacan lo fundamental de la información... (8)

\section{CONCLUSIONES}

Los resultados obtenidos en estainvestigación nos llevan a inferir que en la prensa vasca y navarra ha habido unaumento de los contenidos sobre salud y medicina en la primera mitad del siglo XXI. Así, a pesar de la crisis económica, la salud se ha consolidado como un tema en la agenda de la prensa vasca y navarra.

A pesarde este aumento en la cantidad, hay aspectos de su cobertura informativa que podrían mejorarse para queel espacio dedicado a la salud por los diarios se equipare a la relevancia social de este tema:

En primer lugar, la gran mayoría de las informaciones publicadas no han recibido la suficiente relevancia como para aparecer en las portadas de los diarios. Además, son textos que encuentran su espacio en las páginas interiores en secciones muy poco específicas como Sociedad o Local y lo hacen mayoritariamente en forma de noticia y noticia breve. Ello nos lleva a concluir que la prensa vasca y navarra aborda los temas de salud desde una perspectiva informativa, muy ligada a la actualidad diaria y que no realiza un tratamiento en profundidad de los temas.

Del mismo modo, se ha verificado la falta de especialización entre los profesionales que tratan esa información. Ninguno de los periodistas que cubren noticias sobre salud en los diarios analizados está especializado en salud, ya que todos y todas se encargan de la sección de Sociedad, donde tienen cabida múltiples y variados temas. Hay que decir que, aunque los profesionales no cuenten con especialización en el área de la salud y la sanidad, los periodistas entrevistados presentan un interés y una sensibilidad especial hacia el tema.Esta es una realidad habitual en este tipo de diarios locales o comarcales. Aunque estudiosos como Montserrat Quesada defiendan lanecesidad de redactores especializados, en el periodismo científico español los diarios cuentancada día más con redactores generalistas para elaborar este tipo de información. Esta tendencia es más acusada en los medios regionales donde no ven la necesidad de incorporar a sus redacciones periodistas especializados (Elías,2002a: 401).

En tercer lugar, el estudio constata que los temas de salud en la prensa vasca y navarra no plantean un enfoque educativo. Más de tres cuartas partes de las piezas analizadas no cuentan con una perspectiva educativa cuyo propósito sea ampliar el conocimiento de los lectores y lectoras más allá de la mera información del día a día.Sin embargo, los profesionales que elaboran estos contenidos están muy concienciados de la función divulgativa que deben integrar los textos sobre salud. "La divulgación exige una explicación de las causas y circunstancias que concurren en el hecho noticioso, y esto sólo puede conseguirse con una adecuada cultura periodística del redactor" (Elías, 2002a: 393). Además, señalan el esfuerzo que se realiza por parte de los medios y sobre todo de los periodistas que cubren estas informaciones para que los temas contengan un punto 
de vista educativo y de prevención para con los lectores. No obstante, reconocen las limitaciones para llevar a cabo esa función, por estar muy sujetos a la información diaria, a la vorágine del día a día, y no dedicar los recursos humanos necesarios para este fin.

Por último, en la prensa vasca y navarra se emplean fundamentalmente fotografías con poco valor informativo y se prescinde, principalmente por razones económicas, del uso de gráficos e infografías que facilitan la comprensión, la explicación y el enfoque educativo de los temas de salud y sanidad.

\section{REFERENCIAS BIBLIOGRÁFICAS}

ARMENTIA, J.I; y CAMINOS, J.M. (2009): Redacción informativa en prensa. Barcelona: Ariel.

BARDIN, L. (2002): El análisis de contenido. Madrid: Akal.

CAMACHO, I; PEÑAFIEL, C; RONCO, M. (2012): "Necesidad de una información sanitaria de calidad: análisis de las noticias sobre salud en la prensa vasca". Actas del III Congreso Internacional de la Asociación Española de Investigadores de la Comunicación): Comunicación y Riesgo, AE-IC, Tarragona: http://www.aeic2012tarragona.org/comunicacions_cd/ok/170.pdf.

CORTIÑAS, S. (2007): "La globalización del periodismo científico bajo patrones anglosajones: un estudio de caso en la periferia". Estudios sobre el Mensaje Periodístico, n 13, 369-384.

CORTIÑAS, S; LACANO, D; y PONT, C. (2013): "Estudio sobre los efectos de la crisis en la información de ciencia en los medios. ¿Qué opinan los periodistas científicos?”. ZILLESK; CUENCA J; y ROM J. (eds.). (2013): Breaking the Media Value Chain VII International Conference on Communication and Reality. Barcelona: Facultat de Comunicació i Relacions Internacionals Blanquerna, 79-89.

COSTA, C. (2008): "Medicina y salud en la prensa. Las noticias de salud en los principales diarios de Galicia". Revista Latina de Comunicación Social 63, España:

http://www.ull.es/publicaciones/latina/_2008/03/Costa_Sanchez.html.

ECHEGARAY, L; y AYESTARAN, A. (2012): "Base metodológica para el estudio y análisis sobre la información de salud en la prensa diaria vasca y navarra (2001-2010)". Actas del III Congreso Internacional de la Asociación Española de Investigadores de la Comunicación): Comunicación y Riesgo, AE-IC, Tarragona: http://www.aeic2012tarragona.org/esp/abstracts3.asp?id_seccion=1\&id_slot=4

ELIAS, C. (2002a): "Periodistas especializados en ciencia: formación, reconocimiento e influencia". Mediatika. 8, 389-403.

ELIAS, C. (2002b): "Influencia de las revistas de impacto en el periodismo científico y en la ciencia actual". Reis. Revista Española de Investigaciones Sociológicas, núm. 98, 123-137.

ELIAS, C. (2003): "Los suplementos especializados como guetos de noticias en la prensa generalista: el caso de los científicos y sanitarios”. Ámbitos. No especial 9-10, 171-186.

FOX, S. (2011): Health Topics. Washington, DC: Pew Research Center. http://www.pewinternet.org/ /media//Files/Reports/2011/PIP_Health_Topics.pdf

MORENO, C. (2003): "La investigación universitaria en periodismo científico". Ámbitos, No especial 9-10, 121141.

MORENO, C. (2010): "La construcción periodística de la ciencia a través de los medios de comunicación social: hacia una taxonomía de la difusión del conocimiento científico". ArtefaCToS Vol. 3, n. ${ }^{\circ}$, 109-130.

NELKIN, D. (2001): "Beyond Risk: Reporting about Genetics in the Post-Asilomar Press", Perspectives in Biology and Medicine, vol. 44, $n^{\circ}$ 2, 199-207.(DOI: 10.1353/pbm.2001.0032). 
OBSERVATORIO DE LA COMUNICACIÓN CIENTÍFICA; FUNDACIÓN VILA CASAS. (2006): Informe Quiral 2005. Medicina, comunicación y sociedad. Barcelona: Rubes.

OBSERVATORIO DE LA COMUNICACIÓN CIENTÍFICA; FUNDACIÓN VILA CASAS. (2008): Informe Quiral 10 años. Medicina y Salud en la prensa diaria. Barcelona: Rubes.

OBSERVATORIO DE LA COMUNICACIÓN CIENTÍFICA; FUNDACIÓN VILA CASAS. (2010): Informe Quiral 2009. Medicina, comunicación y sociedad. Barcelona: Rubes.

OBSERVATORIO DE LA COMUNICACIÓN CIENTÍFICA; FUNDACIÓN VILA CASAS. (2012): Informe Quiral 2011. Cáncer. Medicina, comunicación y sociedad. Barcelona: Rubes.

REVUELTA, G; y MINELLI DE OLIVEIRA, J. (2008): "La salud y la biomedicina en la prensa diaria. Un análisis de diez años". Periodística 11, 55-67. http://www.raco.cat/index.php/Periodistica/article/view/245697/328972

TABAKMAN, R. (2011): La salud en los Medios. Medicina para Periodistas. Periodismo para Médicos. Gran Bretaña: Intenal Medical Publishing.

TAYLOR, S.J; y BOGDAN, R. (1987):Introducción a los métodos cualitativos de investigación: La búsqueda de significados. Barcelona:Paidós Básica.

UGARTE, A; MENÉNDEZ, T; CUESTA, U. (2009): Prensa escrita y salud en Madrid. Una revisión del modelo de la Agenda Setting y su aplicación a la dinámica de las noticias sobre salud en Madrid. Madrid: Universidad Complutense.

http://www.fundacionabbott.es/documentos/publicaciones/comunicacion_y_salud/prensaescritaysaludmadrid.pdf

VALLES, M. (1999): Técnicas cualitativas de investigación social. Reflexión metodológica y práctica profesional. Madrid, Síntesis.

VISWANATH, K. y otros (2008): "Occupational practices and the making of health news: A national survey of US health and medical science journalists". The Journal of Health Communication, vol. $13, n^{\circ} 8,759-777$. (DOI: 10.1080/10810730802487430).

WIMMER, R; DOMINICK, J.R. (1996): La investigación científica de los medios de comunicación. Una introducción a sus métodos. Barcelona: Bosch.

YOUNG, E;NORMAN, R; y HUMPHREYS, R. (2008): "Medicine in the Popular Press: The Influence of the Media on Perceptions of Disease", PLOS ONE, vol. 3, $n^{\circ} 10$ : e3552. (DOI: 10.1371/journal.pone.0003552).

ZABALETA, I. (1997): Komunikazioaren eta ikerkuntzako metodología. Bilbao:Udako Euskal Unibersitatea.

\section{Breve semblanza de las autoras:}

Alazne Aiestaran Yarza (Ermua, 1972) es profesora del Departamento de Periodismo de la Universidad del País Vasco. Doctora en Ciencias de la Información por la Universidad del País Vasco (2007). Ha trabajado como directora de una publicación local en euskara durante 7 años. Ha participado en proyectos financiados por la UPV-EHU, Gobierno Vasco y MICINN que tienen reflejo en diversos congresos, artículos y capítulos de libros. Desde 2012 pertenece al grupo de investigación HGH (Medios de Comunicación, Sociedad y Educación en euskara) oficialmente reconocido por la UPV/EHU.

Idoia Camacho Markina (Barakaldo, 1972) es profesora agregada del Departamento de Periodismo de la Universidad del País Vasco. Doctora en Ciencias de la Información por la Universidad del País Vasco (2002). Su labor investigadora se ha desarrollado en torno a la prensa local, a la documentación informativa y, en los últimos años, al periodismo especializado en salud. Dentro de este ámbito, destaca la coordinación de un libro: La especialización en el periodismo: Formarse para informar (2010), además de sus investigaciones sobre periodismo sanitario, que se traducen en varias contribuciones a congresos, artículos científicos y capítulos de 
libros sobre el tema.

María Milagros Ronco López (Salamanca, 1951) es profesora titular del Departamento de Periodismo de la Universidad del País Vasco. Doctora en Ciencias de la Información por la Universidad Complutense de Madrid (2000). Especialista Universitaria en Documentación por el Departamento de Biblioteconomía y Documentación de la Universidad Complutense de Madrid (1998). Ha participado como investigadora en varios Proyectos financiados por la UPV-EHU y por la Comisión Española de la UNESCO como investigadora principal. Autora de varias monografías, capítulos de libro y artículos en torno al tema de la Documentación-Historia, desarrollo, problemáticas, funcionamiento.

(1) Este estudio se enmarca dentro del proyecto de investigación financiado por la Universidad del País Vasco "La información de salud en la prensa diaria vasca (2001-2010)", con código EHU/10/47. El equipo de investigación está compuesto por: IP: Idoia Camacho Markina. Carmen Peñafiel Saiz, Milagros Ronco López, Alazne Aiestaran Yarza profesoras del departamento de periodismo de la UPV/EHU. Lázaro Echegaray Eizaguirre investigador y profesor del centro colaborador Escuela Universitaria de la Cámara de Comercio de Bilbao.

(2) Encuesta no publicada. Datos recogidos en la revista médica JANO.es del 23 de septiembre de 2010, disponible en: http://www.jano.es/jano/ctl_servlet?_f=11\&iditem=11510\&idtabla=1.

(3) Euskaldunon Egunkaria se cerró por orden de la Audiencia Nacional acusado de relación con ETA. En la operación detuvieron a 10 miembros de la dirección del periódico. 7 años más tarde, el 12 de abril de 2010, la Audiencia Nacional absolvió a los cinco directivos que finalmente estaban encausados por pertenencia a ETA. La causa económica sigue pendiente de sentencia en mayo de 2013.

(4) Los periodistas entrevistados fueron: María José Echevarría de Diario de Navarra, Ainara Arratibel de Egunkaria y Berria, Carlos Martínez de Noticias de Álava, Fermín Apezteguia de El Correo y Ana Úrsula Soto de Noticias de Gipuzkoa.

(5) Extracto de la entrevista en profundidad realizada a Fermín Apezteguia del diario El Correo expresamente para esta investigación.

(6) Extracto de la entrevista en profundidad realizada a María José Echeverria del periódico Diario de Navarra expresamente para esta investigación.

(7) Extracto de la entrevista en profundidad realizada a Carlos Martínez del periódico Noticias de Álava expresamente para esta investigación.

(8) Extracto de la entrevista en profundidad realizada a Fermín Apezteguia del diario El Correo expresamente para esta investigación.

Ámbitos. Revista Internacional de Comunicación, n.26, año 2014, tercer trimestre (otoño).

Recibido: $31 / 3 / 2014$

Aprobado: 28/4/2014 